\title{
Article
}

\section{A New Modified Fixed-Point Iteration Process}

\author{
Chanchal Garodia ${ }^{1}$, Afrah A. N. Abdou ${ }^{2,3}$ and Izhar Uddin 1,*(D) \\ 1 Department of Mathematics, Jamia Millia Islamia, New Delhi 110025, India; c.garodia85@gmail.com \\ 2 Department of Mathematics, Faculty of Sciences, King Abdulaziz University, Jeddah 21589, Saudi Arabia; \\ aabdou@kau.edu.sa or aabdou@uj.edu.sa \\ 3 Department of Mathematics, Faculty of Sciences, University of Jeddah, Jeddah 23218, Saudi Arabia \\ * Correspondence: izharuddin1@jmi.ac.in
}

check for

updates

Citation: Garodia, C.; Abdou,

A.A.N.; Uddin, I. A New Modified Fixed-Point Iteration Process. Mathematics 2021, 9, 3109. https:// doi.org/10.3390/math9233109

Academic Editor: Christopher Goodrich

Received: 28 October 2021

Accepted: 22 November 2021

Published: 2 December 2021

Publisher's Note: MDPI stays neutral with regard to jurisdictional claims in published maps and institutional affiliations.

Copyright: (c) 2021 by the authors. Licensee MDPI, Basel, Switzerland. This article is an open access article distributed under the terms and conditions of the Creative Commons Attribution (CC BY) license (https:// creativecommons.org/licenses/by/ $4.0 /)$.

\begin{abstract}
In this paper, we present a new modified iteration process in the setting of uniformly convex Banach space. The newly obtained iteration process can be used to approximate a common fixed point of three nonexpansive mappings. We have obtained strong and weak convergence results for three nonexpansive mappings. Additionally, we have provided an example to support the theoretical proof. In the process, several relevant results are improved and generalized.
\end{abstract}

Keywords: nonexpansive mapping; common fixed point; iteration process; strong and weak convergence

MSC: 47H09; 47H10

\section{Introduction}

Nonlinear analysis is a natural mixture of Topology, Analysis and Linear Algebra. Fixed-point theory is a very challenging and rapidly growing area of nonlinear functional analysis. Obviously, results dealing with the existence of fixed points are termed as fixed point theorems. Such theorems are very important tools for proving the existence and uniqueness of the solutions to various mathematical models representing phenomena arising in different fields such as: optimization theories, variational inequalities, equilibrium problems, economic theories, chemical equations, neutron transport theory, epidemics and flow of fluids besides facilitating existence and uniqueness theories of differential, integral and partial differential equations etc. Historically, the origin of fixed point theorem is attributed to the work on differential equations by the French mathematicians $\mathrm{H}$. Poincare and Emile Picard. At the end of the 19th century, this theorem was moulded into several successive versions. The general case was first proved in 1910 by Jacques Hadamard and then in 1912 by Luitzen Egbertus Jan Brouwer [1]. Fixed-point theory is relatively old but still a young area of research. There exists a vast literature on fixed-point theory and this is still growing [2-6]. Banach Contraction Principle [7] is one of the prime results of fixed-point theory. The early findings in fixed-point theory revolve around generalization of Banach Contraction Principle. The entire mathematics community had to wait for the first fixed-point theorem for nonexpansive mapping for 43 years. Let $J$ be a nonempty closed convex subset of a uniformly convex Banach space $P$. Then, a mapping $G: J \rightarrow J$ is said to be nonexpansive if $\|G c-G b\| \leq\|c-b\|$ for all $c, b \in J$. A point $c \in J$ is said to be a fixed point of $G$ if $G c=c$. We will denote the set of fixed points of $G$ by $F(G)$. $G$ is called quasi-nonexpansive if $F(G) \neq \varnothing$ and $\|G c-e\| \leq\|c-e\|$ for all $c \in J$ with $e \in F(G)$. It is well known that every nonexpansive mapping with a fixed point is quasi-nonexpansive mapping. It is well known Banach Contraction Principle does not hold good for nonexpansive mappings i.e. nonexpansive mapping need not admit a fixed point on complete metric space. Additionally, Picard iteration need not be convergent for a nonexpansive map in a complete metric space. This led to the beginning of a new era of fixed-point theory for 
nonexpansive mappings using geometric properties. In 1965, Browder [8], Göhde [9] and Kirk [10] gave three basic existence results in respect of nonexpansive mappings.

Approximation of fixed points in different domains for nonlinear mappings using the different iterative processes is the thrust of fixed-point theory. Owing to its importance, fixed-point theory is attracting young researchers across the world and in the last few years many iterative processes have been obtained in different domains. To name a few, we have Mann iteration [11], Ishikawa iteration [12], Halpern iteration [13], Noor iteration [14], S-iteration [15] and Abbas et al. iteration process [16]. In 2016, Thakur et al. [17] obtained a new iteration process and proved some convergence results. The authors also claimed that their process converges faster than Picard, Mann, Ishikawa, Noor, S and Abbas et al. iteration process. In order to define their iteration, let $G: J \rightarrow J$ be a nonexpansive mapping, then a sequence $\left\{c_{n}\right\}$ is constructed from arbitrary $c_{1} \in J$ by:

$$
\left\{\begin{array}{lll}
a_{n} & =\left(1-\kappa_{n}\right) c_{n}+\kappa_{n} G c_{n} & \\
b_{n} & =\left(1-\delta_{n}\right) a_{n}+\delta_{n} G a_{n} \\
c_{n+1} & =\left(1-\mu_{n}\right) G a_{n}+\mu_{n} G b_{n}, \quad n \in \mathbb{N}
\end{array}\right.
$$

where $\left\{\mu_{n}\right\},\left\{\delta_{n}\right\}$ and $\left\{\kappa_{n}\right\}$ are real sequences in $(0,1)$.

Motivated and inspired by the research going on in this direction, we introduce a new iteration process for approximating common fixed point of three nonexpansive mappings. Let $G_{1}, G_{2}, G_{3}: J \rightarrow J$ be three nonexpansive mappings, then the sequence $\left\{c_{n}\right\}$ is generated iteratively by $c_{1} \in J$ and

$$
\left\{\begin{array}{lll}
a_{n} & =\left(1-\kappa_{n}\right) c_{n}+\kappa_{n} G_{1} c_{n} & \\
b_{n}=\left(1-\delta_{n}\right) a_{n}+\delta_{n} G_{2} a_{n} & \\
c_{n+1}=\left(1-\mu_{n}\right) G_{2} a_{n}+\mu_{n} G_{3} b_{n}, & n \in \mathbb{N}
\end{array}\right.
$$

where $\left\{\mu_{n}\right\},\left\{\delta_{n}\right\}$ and $\left\{\kappa_{n}\right\}$ are real sequences in $(0,1)$.

The aim of this paper is to prove some weak and strong convergence results involving the iteration process (2) for three nonexpansive mappings. Furthermore, we provide a numerical example to support our theoretical claims. Our results extend the corresponding results of [17].

\section{Preliminaries}

For making our paper self-contained, we collect some basic definitions and needed results.

Definition 1. A Banach space $P$ is said to be uniformly convex if for each $\epsilon \in(0,2]$ there is a $\alpha>0$ such that for $c, b \in P$ with $\|c\| \leq 1,\|b\| \leq 1$ and $\|c-b\|>\epsilon$, we have

$$
\left\|\frac{c+b}{2}\right\|<1-\alpha
$$

Definition 2. A Banach space $P$ is said to satisfy the Opial's condition if for any sequence $\left\{c_{n}\right\}$ in $P$ which converges weakly to $c \in P$ i.e., $c_{n} \rightarrow c$ implies that

$$
\limsup _{n \rightarrow \infty}\left\|c_{n}-c\right\|<\limsup _{n \rightarrow \infty}\left\|c_{n}-b\right\|
$$

for all $b \in P$ with $b \neq c$.

Examples of Banach spaces satisfying this condition are Hilbert spaces and all $l^{p}$ spaces $(1<p<\infty)$. On the other hand, $L^{p}[0,2 \pi]$ with $1<p \neq 2$ fail to satisfy Opial's condition.

A mapping $G: J \rightarrow P$ is demiclosed at $b \in P$ if for each sequence $\left\{c_{n}\right\}$ in $J$ and each $c \in P, c_{n} \rightarrow c$ and $G c_{n} \rightarrow b$ imply that $c \in J$ and $G c=b$. 
Let $J$ be a nonempty closed convex subset of a Banach $P$, and let $\left\{c_{n}\right\}$ be a bounded sequence in $P$. For $c \in P$ write:

$$
r\left(c,\left\{c_{n}\right\}\right)=\limsup _{n \rightarrow \infty} d\left(c, c_{n}\right) .
$$

The asymptotic radius of $\left\{c_{n}\right\}$ relative to $J$ is given by

$$
r\left(\left\{c_{n}\right\}\right)=\inf \left\{r\left(c, c_{n}\right): c \in J\right\}
$$

and the asymptotic center $A\left(J,\left\{c_{n}\right\}\right)$ of $\left\{c_{n}\right\}$ is defined as:

$$
A\left(J,\left\{c_{n}\right\}\right)=\left\{c \in J: r\left(c,\left\{c_{n}\right\}\right)=r\left(\left\{c_{n}\right\}\right)\right\} .
$$

It is known that in a uniformly convex Banach space, $A\left(J,\left\{c_{n}\right\}\right)$ consists of exactly one point.

Next, we list a lemma which will be useful in our subsequent discussion.

Lemma 1 ([8]). Let J be a nonempty closed convex subset of a uniformly convex Banach space $P$ and $G$ a nonexpansive mapping on $J$. Then, $I-G$ is demiclosed at zero.

The following lemma due to Schu [18] is very useful in our subsequent discussion.

Lemma 2. Let $P$ be a uniformly convex Banach space and $\left\{t_{n}\right\}$ be any sequence such that $0<$ $p \leq t_{n} \leq q<1$ for some $p, q \in \mathbb{R}$ and for all $n \geq 1$. Let $\left\{c_{n}\right\}$ and $\left\{b_{n}\right\}$ be any two sequences of $P$ such that lim sup $\left\|c_{n}\right\| \leq r$, lim sup $\left\|b_{n}\right\| \leq r$ and lim sup $\left\|t_{n} c_{n}+\left(1-t_{n}\right) b_{n}\right\|=r$ for some $r \geq 0$. Then, $\lim _{n \rightarrow \infty}\left\|c_{n}-b_{n}\right\|=0$.

\section{Convergence Results}

Lemma 3. Let $J$ be a nonempty closed convex subset of a Banach space $P$ and $G_{1}, G_{2}, G_{3}: J \rightarrow J$ be three nonexpansive mappings with $F\left(G_{1}\right) \cap F\left(G_{2}\right) \cap F\left(G_{3}\right) \neq \varnothing$. Let $\left\{c_{n}\right\}$ be defined by the iteration process (1.2). Then

(i) $\lim _{n \rightarrow \infty}\left\|c_{n}-e\right\|$ exists for all $e \in F\left(G_{1}\right) \cap F\left(G_{2}\right) \cap F\left(G_{3}\right)$,

(ii) $\lim _{n \rightarrow \infty}\left\|G_{1} c_{n}-c_{n}\right\|=\lim _{n \rightarrow \infty}\left\|G_{2} c_{n}-c_{n}\right\|=\lim _{n \rightarrow \infty}\left\|G_{3} c_{n}-c_{n}\right\|=0$.

Proof. (i) Let $e \in F\left(G_{1}\right) \cap F\left(G_{2}\right) \cap F\left(G_{3}\right)$. Then, using (2) we get

$$
\begin{aligned}
\left\|a_{n}-e\right\| & =\left\|\left(1-\kappa_{n}\right) c_{n}+\kappa_{n} G_{1} c_{n}-e\right\| \\
& \leq\left(1-\kappa_{n}\right)\left\|c_{n}-e\right\|+\kappa_{n}\left\|G_{1} c_{n}-e\right\| \\
& \leq\left(1-\kappa_{n}\right)\left\|c_{n}-e\right\|+\kappa_{n}\left\|c_{n}-e\right\| \\
& =\left\|c_{n}-e\right\|
\end{aligned}
$$

and

$$
\begin{aligned}
\left\|b_{n}-e\right\| & \left.=\|\left(1-\delta_{n}\right) a_{n}+\delta n G_{2} a_{n}\right)-e \| \\
& \leq\left(1-\delta_{n}\right)\left\|a_{n}-e\right\|+\delta_{n}\left\|G_{2} a_{n}-e\right\| \\
& \leq\left(1-\delta_{n}\right)\left\|a_{n}-e\right\|+\delta_{n}\left\|a_{n}-e\right\| \\
& =\left\|a_{n}-e\right\| \\
& \leq\left\|c_{n}-e\right\| .
\end{aligned}
$$

Using (3) and (4) we obtain

$$
\begin{aligned}
\left\|c_{n+1}-e\right\| & =\left\|\left(1-\mu_{n}\right) G_{2} a_{n}+\mu_{n} G_{3} b_{n}-e\right\| \\
& \leq\left(1-\mu_{n}\right)\left\|G_{2} a_{n}-e\right\|+\mu_{n}\left\|G_{3} b_{n}-e\right\| \\
& \leq\left(1-\mu_{n}\right)\left\|a_{n}-e\right\|+\mu_{n}\left\|b_{n}-e\right\| \\
& \leq\left\|a_{n}-e\right\| \\
& \leq\left\|c_{n}-e\right\| .
\end{aligned}
$$


Thus, $\left\{\left\|c_{n}-e\right\|\right\}$ is bounded and non-increasing for all $e \in F\left(G_{1}\right) \cap F\left(G_{2}\right) \cap F\left(G_{3}\right)$ which gives that $\lim _{n \rightarrow \infty}\left\|c_{n}-e\right\|$ exists for all $e \in F\left(G_{1}\right) \cap F\left(G_{2}\right) \cap F\left(G_{3}\right)$.

(ii) Let

$$
\lim _{n \rightarrow \infty}\left\|c_{n}-e\right\|=r
$$

From (3) and (4), we obtain

$$
\left\|c_{n+1}-e\right\| \leq\left\|a_{n}-e\right\| \leq\left\|c_{n}-e\right\|
$$

which gives

$$
\lim _{n \rightarrow \infty}\left\|a_{n}-e\right\|=r .
$$

Additionally, Since $G_{1}$ is nonexpansive so, we have

$$
\limsup _{n \rightarrow \infty}\left\|G_{1} c_{n}-e\right\| \leq \limsup _{n \rightarrow \infty}\left\|c_{n}-e\right\|=r .
$$

Now, (6)-(8) along with Lemma 2 gives

$$
\lim _{n \rightarrow \infty}\left\|G_{1} c_{n}-c_{n}\right\|=0
$$

From (4) and (7), we have

$$
\underset{n \rightarrow \infty}{\limsup }\left\|b_{n}-e\right\| \leq \limsup _{n \rightarrow \infty}\left\|a_{n}-e\right\|=r .
$$

Now, consider

$$
\begin{aligned}
\left\|c_{n+1}-e\right\| & \leq\left(1-\mu_{n}\right)\left\|a_{n}-e\right\|+\mu_{n}\left\|b_{n}-e\right\| \\
& \leq\left(1-\mu_{n}\right)\left\|c_{n}-e\right\|+\mu_{n}\left\|b_{n}-e\right\|
\end{aligned}
$$

which gives

$$
\left\|c_{n}-e\right\| \leq\left\|b_{n}-e\right\|+\frac{1}{\mu_{n}}\left(\left\|c_{n}-e\right\|-\left\|c_{n+1}-e\right\|\right) .
$$

Therefore, we get

$$
r \leq \liminf _{n \rightarrow \infty}\left\|b_{n}-e\right\| .
$$

On using (10) and (11), we obtain

$$
\lim _{n \rightarrow \infty}\left\|b_{n}-e\right\|=r .
$$

Additionally, nonexpansiveness of $G_{2}$ and (7) yields

$$
\limsup _{n \rightarrow \infty}\left\|G_{2} a_{n}-e\right\| \leq r .
$$

From (7), (12), (13) and Lemma 2 we obtain

$$
\lim _{n \rightarrow \infty}\left\|G_{2} a_{n}-a_{n}\right\|=0
$$

Consider

$$
\begin{aligned}
\left\|a_{n}-c_{n}\right\| & =\left\|\left(1-\kappa_{n}\right) c_{n}+\kappa_{n} G_{1} c_{n}-c_{n}\right\| \\
& =\kappa_{n}\left\|G_{1} c_{n}-c_{n}\right\| .
\end{aligned}
$$

which on using (9) results into

$$
\lim _{n \rightarrow \infty}|| a_{n}-c_{n} \|=0
$$

Now,

$$
\begin{aligned}
\left\|G_{2} c_{n}-c_{n}\right\| & \leq\left\|G_{2} c_{n}-G_{2} a_{n}\right\|+\left\|G_{2} a_{n}-a_{n}\right\|+\left\|a_{n}-c_{n}\right\| \\
& \leq\left\|G_{2} a_{n}-a_{n}\right\|+2\left\|a_{n}-c_{n}\right\|
\end{aligned}
$$


which on using (14) and (15) yields

$$
\lim _{n \rightarrow \infty}\left\|G_{2} c_{n}-c_{n}\right\|=0
$$

Now, since $G_{3}$ is nonexpansive, so we $h$

$$
\limsup _{n \rightarrow \infty}\left\|G_{3} b_{n}-e\right\| \leq \limsup _{n \rightarrow \infty}\left\|b_{n}-e\right\|=r .
$$

On using, (6), (13), (17) and Lemma 2, we obtain

$$
\lim _{n \rightarrow \infty}\left\|G_{2} a_{n}-G_{3} b_{n}\right\|=0
$$

Additionally,

$$
\begin{aligned}
\left\|b_{n}-c_{n}\right\| & =\left\|\left(1-\delta_{n}\right) a_{n}+\delta_{n} G_{2} a_{n}-c_{n}\right\| \\
& \leq\left(1-\delta_{n}\right)\left\|a_{n}-c_{n}\right\|+\delta_{n}\left(\left\|G_{2} a_{n}-a_{n}\right\|+\left\|a_{n}-c_{n}\right\|\right)
\end{aligned}
$$

which along with (14) and (15) results into

$$
\lim _{n \rightarrow \infty}|| b_{n}-c_{n} \|=0
$$

Now

$$
\begin{aligned}
\left\|G_{3} c_{n}-c_{n}\right\| & \leq\left\|G_{3} c_{n}-G_{3} b_{n}\right\|+\left\|G_{3} b_{n}-G_{2} a_{n}\right\|+\left\|G_{2} a_{n}-a_{n}\right\|+\left\|a_{n}-c_{n}\right\| \\
& \leq\left\|c_{n}-b_{n}\right\|+\left\|G_{3} b_{n}-G_{2} a_{n}\right\|+\left\|G_{2} a_{n}-a_{n}\right\|+\left\|a_{n}-c_{n}\right\|
\end{aligned}
$$

on using (14), (15), (18) and (19), we obtain

$$
\lim _{n \rightarrow \infty}\left\|G_{3} c_{n}-c_{n}\right\|=0
$$

Now, we prove the weak convergence of iteration process (2) using Opial property.

Theorem 1. Let J be a nonempty closed convex subset of a uniformly convex Banach space $P$ which satisfies the Opial's condition and $G_{1}, G_{2}, G_{3}: J \rightarrow J$ be three nonexpansive mapping with $F\left(G_{1}\right) \cap F\left(G_{2}\right) \cap F\left(G_{3}\right) \neq \varnothing$. If $\left\{c_{n}\right\}$ is defined by the iteration process (2), then $\left\{c_{n}\right\}$ converges weakly to a common fixed point of $G_{1}, G_{2}$ and $G_{3}$.

Proof. Let $e \in F\left(G_{1}\right) \cap F\left(G_{2}\right) \cap F\left(G_{3}\right)$. Then, from Lemma $3 \lim _{n \rightarrow \infty}\left\|c_{n}-e\right\|$ exists. In order to show the weak convergence of the iteration process (2) to a common fixed point of $G_{1}, G_{2}$ and $G_{3}$, we will prove that $\left\{c_{n}\right\}$ has a unique weak subsequential limit in $F\left(G_{1}\right) \cap F\left(G_{2}\right) \cap F\left(G_{3}\right)$. For this, let $\left\{c_{n_{j}}\right\}$ and $\left\{c_{n_{k}}\right\}$ be two subsequences of $\left\{c_{n}\right\}$ which converges weakly to $w$ and $u$ respectively. By Lemma 3, we have $\lim _{n \rightarrow \infty}\left\|G_{1} c_{n}-c_{n}\right\|=$ $\lim _{n \rightarrow \infty}\left\|G_{2} c_{n}-c_{n}\right\|=\lim _{n \rightarrow \infty}\left\|G_{3} c_{n}-c_{n}\right\|=0$ and using the Lemma 1, we have $I-G_{1}, I-G_{2}$ and $I-G_{3}$ are demiclosed at zero. So $w, u \in F\left(G_{1}\right) \cap F\left(G_{2}\right) \cap F\left(G_{3}\right)$. 
Next, we show the uniqueness. Since $w, u \in F\left(G_{1}\right) \cap F\left(G_{2}\right) \cap F\left(G_{3}\right)$, so $\lim _{n \rightarrow \infty}\left\|c_{n}-w\right\|$ and $\lim _{n \rightarrow \infty}\left\|c_{n}-u\right\|$ exists. Let $w \neq u$. Then, by Opial's condition, we obtain

$$
\begin{aligned}
\lim _{n \rightarrow \infty}\left\|c_{n}-w\right\| & =\lim _{n \rightarrow \infty}\left\|c_{n_{j}}-w\right\| \\
& <\lim _{n \rightarrow \infty}\left\|c_{n_{j}}-u\right\| \\
& =\lim _{n \rightarrow \infty}\left\|c_{n}-u\right\| \\
& =\lim _{n \rightarrow \infty}\left\|c_{n_{k}}-u\right\| \\
& <\lim _{n \rightarrow \infty}\left\|c_{n_{k}}-w\right\| \\
& =\lim _{n \rightarrow \infty}\left\|c_{n}-w\right\|
\end{aligned}
$$

which is a contradiction, so $w=u$. Thus, $\left\{c_{n}\right\}$ converges weakly to a common fixed point of $G_{1}, G_{2}$ and $G_{3}$.

Next, we establish some strong convergence results for iteration process (2).

Theorem 2. Let $J$ be a nonempty closed convex subset of a uniformly convex Banach space $P$ and $G_{1}, G_{2}, G_{3}: J \rightarrow J$ be three nonexpansive mappings with $F\left(G_{1}\right) \cap F\left(G_{2}\right) \cap F\left(G_{3}\right) \neq \varnothing$. If $\left\{c_{n}\right\}$ is defined by the iteration process (2), then $\left\{c_{n}\right\}$ converges to a point of $F\left(G_{1}\right) \cap F\left(G_{2}\right) \cap F\left(G_{3}\right)$ if and only if $\liminf _{n \rightarrow \infty} d\left(c_{n}, F\left(G_{1}\right) \cap F\left(G_{2}\right) \cap F\left(G_{3}\right)\right)=0$.

Proof. If the sequence $\left\{c_{n}\right\}$ converges to a point $e \in F\left(G_{1}\right) \cap F\left(G_{2}\right) \cap F\left(G_{3}\right)$, then it is obvious that $\liminf _{n \rightarrow \infty} d\left(c_{n}, F\left(G_{1}\right) \cap F\left(G_{2}\right) \cap F\left(G_{3}\right)\right)=0$.

For the converse part, assume that $\liminf _{n \rightarrow \infty} d\left(c_{n}, F\left(G_{1}\right) \cap F\left(G_{2}\right) \cap F\left(G_{3}\right)\right)=0$. From Lemma 3, we have $\lim _{n \rightarrow \infty}\left\|c_{n}-e\right\|$ exists for all $e \in F\left(G_{1}\right) \cap F\left(G_{2}\right) \cap F\left(G_{3}\right)$, which gives

$$
\left\|c_{n+1}-e\right\| \leq\left\|c_{n}-e\right\| \text { for any } e \in F\left(G_{1}\right) \cap F\left(G_{2}\right) \cap F\left(G_{3}\right)
$$

which yields

$$
d\left(c_{n+1}, F\left(G_{1}\right) \cap F\left(G_{2}\right) \cap F\left(G_{3}\right)\right) \leq d\left(c_{n}, F\left(G_{1}\right) \cap F\left(G_{2}\right) \cap F\left(G_{3}\right)\right) .
$$

Thus, $\left\{d\left(c_{n}, F\left(G_{1}\right) \cap F\left(G_{2}\right) \cap F\left(G_{3}\right)\right)\right\}$ forms a decreasing sequence which is bounded below by zero as well, so we obtain $\lim _{n \rightarrow \infty} d\left(c_{n}, F\left(G_{1}\right) \cap F\left(G_{2}\right) \cap F\left(G_{3}\right)\right)$ exists. As, $\liminf _{n \rightarrow \infty}$ $d\left(c_{n}, F\left(G_{1}\right) \cap F\left(G_{2}\right) \cap F\left(G_{3}\right)\right)=0$ so $\lim _{n \rightarrow \infty} d\left(c_{n}, F\left(G_{1}\right) \cap F\left(G_{2}\right) \cap F\left(G_{3}\right)\right)=0$.

Next, we prove that $\left\{c_{n}\right\}$ is a Cauchy sequence in $J$. Let $\epsilon>0$ be arbitrarily chosen. Since $\lim _{n \rightarrow \infty} d\left(c_{n}, F\left(G_{1}\right) \cap F\left(G_{2}\right) \cap F\left(G_{3}\right)\right)=0$, there exists $n_{0}$ such that for all $n \geq n_{0}$, we have

$$
d\left(c_{n}, F\left(G_{1}\right) \cap F\left(G_{2}\right) \cap F\left(G_{3}\right)\right)<\frac{\epsilon}{4} .
$$

In particular,

$$
\inf \left\{\left\|c_{n_{0}}-e\right\|: e \in F\left(G_{1}\right) \cap F\left(G_{2}\right) \cap F\left(G_{3}\right)\right\}<\frac{\epsilon}{4},
$$

so there must exist a $b \in F\left(G_{1}\right) \cap F\left(G_{2}\right) \cap F\left(G_{3}\right)$ such that

$$
\left\|c_{n_{0}}-b\right\|<\frac{\epsilon}{2}
$$

Thus, for $m, n \geq n_{0}$, we have

$$
\left\|c_{n+m}-c_{n}\right\| \leq\left\|c_{n+m}-b\right\|+\left\|c_{n}-b\right\|<2\left\|c_{n_{0}}-b\right\|<2 \frac{\epsilon}{2}=\epsilon
$$


which shows that $\left\{c_{n}\right\}$ is a Cauchy sequence. Since $J$ is a closed subset of a Banach space $P$, therefore $\left\{c_{n}\right\}$ must converge in $J$. Let, $\lim _{n \rightarrow \infty} c_{n}=s$ for some $s \in J$. Now using $\lim _{n \rightarrow \infty}\left\|G_{1} c_{n}-c_{n}\right\|=0$, we obtain

$$
\begin{aligned}
\left\|s-G_{1} s\right\| & \leq\left\|s-c_{n}\right\|+\left\|c_{n}-G_{1} c_{n}\right\|+\left\|G_{1} c_{n}-G_{1} s\right\| \\
& \leq\left\|s-c_{n}\right\|+\left\|c_{n}-G_{1} c_{n}\right\|+\left\|c_{n}-s\right\| \\
& \rightarrow 0 \text { as } n \rightarrow \infty
\end{aligned}
$$

and hence $s=G_{1} s$. Similarly, we can show that $s=G_{2} s$ and $s=G_{3} s$, thus $s \in F\left(G_{1}\right) \cap$ $F\left(G_{2}\right) \cap F\left(G_{3}\right)$. This proves our result.

Three mappings $G_{1}, G_{2}, G_{3}: J \rightarrow J$ are said to satisfy the Condition (A) ([19]) if there exists a nondecreasing function $t:[0, \infty) \rightarrow[0, \infty)$ with $t(0)=0$ and $t(r)>0$ for all $r \in(0, \infty)$ such that

$$
\left\|u-G_{1} u\right\| \geq t\left(d\left(u, F\left(G_{1}\right) \cap F\left(G_{2}\right) \cap F\left(G_{3}\right)\right)\right)
$$

or

$$
\left\|u-G_{2} u\right\| \geq t\left(d\left(u, F\left(G_{1}\right) \cap F\left(G_{2}\right) \cap F\left(G_{3}\right)\right)\right)
$$

or

$$
\left\|u-G_{3} u\right\| \geq t\left(d\left(u, F\left(G_{1}\right) \cap F\left(G_{2}\right) \cap F\left(G_{3}\right)\right)\right)
$$

for all $u \in J$.

Theorem 3. Let $J$ be a nonempty closed convex subset of a uniformly convex Banach space P. Let $G_{1}, G_{2}, G_{3}: J \rightarrow J$ be three nonexpansive mapping such that $F\left(G_{1}\right) \cap F\left(G_{2}\right) \cap F\left(G_{3}\right) \neq \varnothing$ and $\left\{c_{n}\right\}$ be the sequence defined by (2). If $G_{1}, G_{2}$ and $G_{3}$ satisfies Condition $(A)$, then $\left\{c_{n}\right\}$ converges strongly to a point of $F\left(G_{1}\right) \cap F\left(G_{2}\right) \cap F\left(G_{3}\right)$.

Proof. From (20) we obtain $\lim _{n \rightarrow \infty} d\left(c_{n}, F\left(G_{1}\right) \cap F\left(G_{2}\right) \cap F\left(G_{3}\right)\right)$ exists.

Additionally, by Lemma 3, we have $\lim _{n \rightarrow \infty}\left\|c_{n}-G_{1} c_{n}\right\|=\lim _{n \rightarrow \infty}\left\|c_{n}-G_{2} c_{n}\right\|=\lim _{n \rightarrow \infty} \| c_{n}-$ $G_{3} c_{n} \|=0$.

It follows from condition (A) that

$$
\lim _{n \rightarrow \infty} t\left(d\left(c_{n}, F\left(G_{1}\right) \cap F\left(G_{2}\right) \cap F\left(G_{3}\right)\right)\right) \leq \lim _{n \rightarrow \infty}\left\|c_{n}-G_{1} c_{n}\right\|=0,
$$

or

$$
\lim _{n \rightarrow \infty} t\left(d\left(c_{n}, F\left(G_{1}\right) \cap F\left(G_{2}\right) \cap F\left(G_{3}\right)\right)\right) \leq \lim _{n \rightarrow \infty}\left\|c_{n}-G_{2} c_{n}\right\|=0,
$$

or

$$
\lim _{n \rightarrow \infty} t\left(d\left(c_{n}, F\left(G_{1}\right) \cap F\left(G_{2}\right) \cap F\left(G_{3}\right)\right)\right) \leq \lim _{n \rightarrow \infty}\left\|c_{n}-G_{3} c_{n}\right\|=0,
$$

so that $\lim _{n \rightarrow \infty} t\left(d\left(c_{n}, F\left(G_{1}\right) \cap F\left(G_{2}\right) \cap F\left(G_{3}\right)\right)\right)=0$.

Since $t$ is a nondecreasing function satisfying $t(0)=0$ and $t(r)>0$ for all $r \in(0, \infty)$, therefore $\lim _{n \rightarrow \infty} d\left(c_{n}, F\left(G_{1}\right) \cap F\left(G_{2}\right) \cap F\left(G_{3}\right)\right)=0$. $F\left(G_{3}\right)$.

By Theorem 2, the sequence $\left\{c_{n}\right\}$ converges strongly to a point of $F\left(G_{1}\right) \cap F\left(G_{2}\right) \cap$

\section{Numerical Example}

In this section, with the help of an example we will show the convergence of (1.2) to common fixed point of three nonexpansive mappings. 
Example 1. Let $P=\mathbb{R}$ with the usual norm, $J=[0, \infty)$ and $G_{1}, G_{2}, G_{3}: J \rightarrow J$ be defined as: $G_{1}=\frac{c}{3}, G_{2}=\frac{c}{4}$ and $G_{3}=\frac{c}{5}$.

It is very easy to show that $G_{1}, G_{2}$ and $G_{3}$ are nonexpansive mappings and 0 is their common fixed point. Set $\mu_{n}=\frac{1}{3 n+7}, \delta_{n}=\frac{2 n}{5 n+2}$ and $\kappa_{n}=\frac{n}{n+2}$. We obtain the following tables and graphs for different initial values.

It is evident from below Tables 1 and 2, Figures 1 and 2 that our Algorithm (1.2) converges efficiently to common fixed point of three above mentioned nonexpansive mappings.

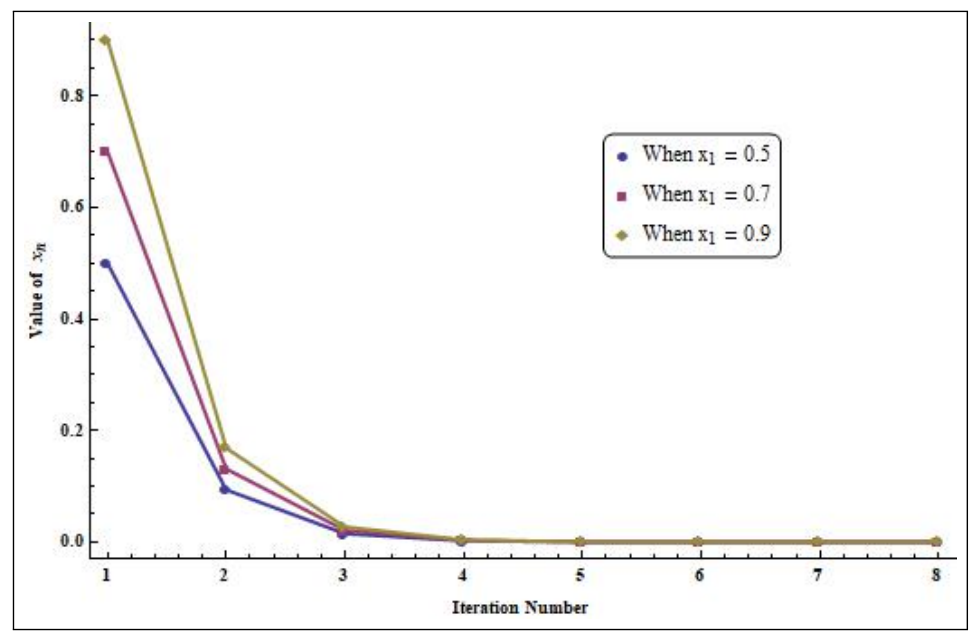

Figure 1. Graph corresponding to Table 1.

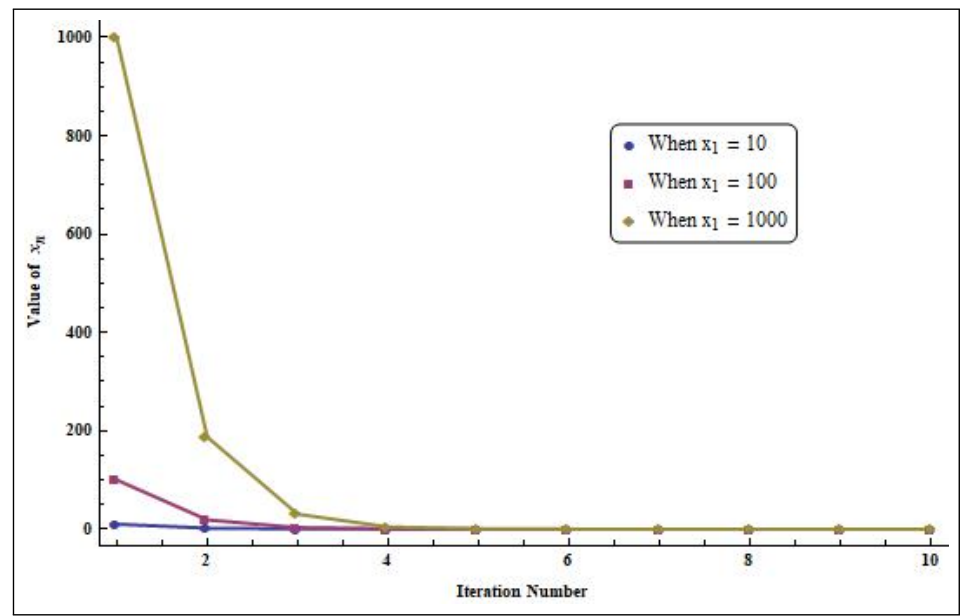

Figure 2. Graph corresponding to Table 2.

Table 1. Values of the iteration corresponding different initial values near 0 .

\begin{tabular}{cccc}
\hline Step & When $\boldsymbol{c}_{\mathbf{1}}=\mathbf{0 . 5}$ & When $\boldsymbol{c}_{\mathbf{1}}=\mathbf{0 . 7}$ & When $\boldsymbol{c}_{\mathbf{1}}=\mathbf{0 . 9}$ \\
\hline 1 & 0.5 & 0.7 & 0.9 \\
2 & 0.09361111 & 0.1310556 & 0.1685 \\
3 & 0.01512179 & 0.02117051 & 0.02721923 \\
4 & 0.002209895 & 0.003093853 & 0.00397781 \\
5 & 0.0003001744 & 0.0004202442 & 0.000540314 \\
6 & 0.00003855415 & 0.00005397581 & 0.00006939747 \\
7 & $4.737341 \times 10^{-6}$ & $6.632277 \times 10^{-6}$ & $8.527214 \times 10^{-6}$ \\
8 & $5.615388 \times 10^{-7}$ & $7.861544 \times 10^{-7}$ & $1.01077 \times 10^{-6}$ \\
\hline
\end{tabular}


Table 2. Values of the iteration corresponding different initial values which is away from 0 .

\begin{tabular}{cccc}
\hline Step & When $\boldsymbol{c}_{\mathbf{1}}=\mathbf{1 0}$ & When $\boldsymbol{c}_{\mathbf{1}}=\mathbf{1 0 0}$ & When $\boldsymbol{c}_{\mathbf{1}}=\mathbf{1 0 0 0}$ \\
\hline 1 & 10 & 100 & 1000 \\
2 & 1.872222 & 18.72222 & 187.2222 \\
3 & 0.3024359 & 3.024359 & 30.24359 \\
4 & 0.04419789 & 0.44197897 & 4.419789 \\
5 & 0.006003488 & 0.06003488 & 0.6003488 \\
6 & 0.000771083 & 0.00771083 & 70.0771083 \\
7 & 0.00009474682 & 0.0009474682 & 0.009474682 \\
8 & 0.00001123078 & 0.0001123078 & 0.001123078 \\
9 & $1.292143 \times 10^{-6}$ & 0.00001292143 & 0.0001292143 \\
10 & $1.449783 \times 10^{-7}$ & $1.449783 \times 10^{-6}$ & 0.00001449783 \\
\hline
\end{tabular}

\section{Conclusions}

A new iteration process is proposed in the framework of uniformly convex Banach space dealing with the common fixed point of three nonexpansive mappings. Some convergence results are also proved along with a numerical example. It should be noted that $G_{1}=G_{2}=G_{3}=G$ is a special case of (2), so our main results extend the results of [17] from one nonexpansive mapping to three nonexpansive mappings.

Author Contributions: Conceptualization, I.U.; Formal analysis, C.G.; Funding acquisition, A.A.N.A.; Investigation, C.G.; Methodology, C.G. and I.U.; Supervision, A.A.N.A. and I.U.; Validation, C.G.; Writing-original draft, C.G.; Writing-review and editing, A.A.N.A. and I.U. All authors have read and agreed to the published version of the manuscript.

Funding: Deanship of Scientific Research (DSR), King Abdulaziz University, Jeddah, grant No. D-051-363-1440.

Institutional Review Board Statement: Not applicable.

Informed Consent Statement: Not applicable.

Acknowledgments: The authors are very grateful to the anonymous referees for. This project was funded by the Deanship of Scientific Research (DSR), King Abdulaziz University, Jeddah, under grant No. D-051-363-1440. The authors, therefore, gratefully acknowledge DSR technical and financial support.

Conflicts of Interest: The authors declare no conflict of interest.

\section{References}

1. Brouwer, L. Uber Abbildungen von Mannigfaltigkeiten. Math. Ann. 1912, 70, 97-115.

2. Yao, Y.; Leng, L.; Postolache, M.; Zheng, X. Mann-type iteration method for solving the split common fixed point problem. J. Nonlinear Convex Anal. 2017, 18, 875-882.

3. Yao, Y.; Qin, X.; Yao, J.C. Projection methods for firmly type nonexpansive operators. J. Nonlinear Convex Anal. $2018,19,407-415$.

4. Garodia, C.; Uddin, I. Approximating common fixed points of non-expansive mappings in CAT(0) spaces. U.P.B. Sci. Bull. Ser. A 2019, 81, 85-96.

5. Garodia, C.; Uddin, I. A new iterative method for solving split feasibility problem. J. Appl. Anal. Comput. 2020, 10, 986-1004. [CrossRef]

6. Garodia, C.; Uddin, I. A new fixed-point algorithm for finding the solution of a delay differential equation. AIMS Math. 2020, 5, 3182-3200. [CrossRef]

7. Banach, S. Sur les operations dans les ensembles abstraits et leurs applications. Fundam. Math. 1922, 3, 133-181. [CrossRef]

8. Browder, F.E. Nonexpansive nonlinear operators in a Banach space. Proc. Natl. Acad. Sci. USA 1965, 54, 1041-1044. [CrossRef] [PubMed]

9. Göhde, D. Zum Prinzip der kontraktiven abbildung. Math. Nachr. 1965, 30, 251-258. [CrossRef]

10. Kirk, W.A. A fixed point theorem for mappings which do not increase distances. Am. Math. Mon. 1965, 72, 1004-1006. [CrossRef]

11. Mann, W.R. Mean value methods in iteration. Proc. Am. Math. Soc. 1953, 4, 506-510. [CrossRef]

12. Ishikawa, S. Fixed points by a new iteration method. Proc. Am. Math. Soc. 1974, 44, 147-150. [CrossRef]

13. Halpern, B. Fixed points of nonexpanding maps. Bull. Am. Math. Soc. 1967, 73, 957-961. [CrossRef]

14. Noor, M.A. New approximation schemes for general variational inequalities. J. Math. Anal. Appl. 2000, 251, 217-229. [CrossRef] 
15. Regan, R.P.A.D.Ó.; Sahu, D.R. Iterative construction of fixed points of nearly asymptotically nonexpansive mappings. J. Nonlinear Convex Anal. 2007, 8, 61-79.

16. Abbas, M.; Nazir, T. A new faster iteration process applied to constrained minimization and feasibility problems. Mat. Vesn. 2014, $66,223-234$.

17. Thakur, B.S.; Thakur, D.; Postolache, M. A New iteration scheme for approximating fixed points of nonexpansive mappings. Filomat 2016, 30, 2711-2720. [CrossRef]

18. Schu, J. Weak and strong convergence to fixed points of asymptotically nonexpansive mappings. Bull. Aust. Math. Soc. 1991, 43, 153-159. [CrossRef]

19. Senter, H.F.; Dotson, W.G. Approximating fixed points of nonexpansive mappings. Proc. Am. Math. Soc. 1974, 44, 375-380. [CrossRef] 